\title{
Rapid assessment of mycotoxins in wine by on-line SPE-UHPLC-FLD
}

\author{
Alina-Mihaela Nistor ${ }^{1}$, Ştefan-Dragoş Cotan $^{1}$, Constantin-Bogdan Nechita ${ }^{2}$, Alexandru Tarţian ${ }^{1}$, Marius Niculaua $^{2}$, \\ and Valeriu V. Cotea ${ }^{1}$
}

\author{
${ }^{1}$ University of Agricultural Sciences and Veterinary Medicine Iaşi, Romania \\ ${ }^{2}$ Reserch Centre for Oenology, Romanian Academy Iaşi branch, Romania
}

\begin{abstract}
According to the latest statistics, grapes are one of the largest fruit crops worldwide. In this regard, it is important to consider all factors influencing quality of grapes and wine. In the last years, scientist focused on the study of mycotoxins that can influence the quality of wine. It is considered that toxins produced by moulds, causing significant economic losses, affect approximately one quarter of the world grape production. If the selective sorting of infected grapes is not done adequately, wine will present a major risk to consumers, mycotoxins being considered by the "International Agency for Cancer Research" a carcinogenic compound. The main mycotoxins monitored in this study come from Aspergillus sp., and are represented by aflatoxins B1, B2, G1, G2 and ochratoxin A. This study purpose is to develop a faster method for the analysis of mycotoxins, in order to increase rapidity and efficiency for the evaluation of the degree of infestation in wine. The purposed method is using an on-line large volume injection coupled to pre-concentration of sample (SPE) which is directly transfer to the ultra-high-pressure liquid chromatography (UHPLC) column for separation and the detection by means of the fluorescence detector (FLD). As the maximum tolerated level for mycotoxins in wines is $2 \mathrm{ppm}$, this method is able to detect under this limits of quantification with RSD below $2 \%$.
\end{abstract}

\section{Introduction}

Worldwide grapes are one of the largest fruit crops, according to the latest FAO-OIV report from 2016. Each year 75 million tons of grapes are produced, which makes them the most widespread fruit [1]. From this total, it is estimated that approximately a quarter is damaged by mould. Such a large proportion of contamination automatically implies economic loss [2,3]. Grape and wine contamination can take place during two stages: during development or after harvest. In the first stage, the crops can be degraded when insects, birds, animals, hailstone, storms, frost, becoming therefore vulnerable to the onset of mould, physically damage it. Also in the development stage, the crops can suffer from stress due to harsh heat and drought. In the second stage, the contamination might happen if the healthy crops are kept in wet and not airy spaces during transportation, storage or usage. A major contamination risk is when the crops are harvested late and it is caught in the rain or this could happen during harvesting $[4,5]$.

Mycotoxins are byproducts of mould metabolism, from where their name comes (Online Etymology Dictionary). Secondary metabolites are filamentary fungi that are highly toxic for humans and animals alike. Diseases caused by mycotoxin intoxication are generally called mycotoxicoses. Their symptoms depend on the type of mycotoxin, the duration of exposure, the age and gender of the infected person, as well as on the person's health. Some common symptoms are anemia, anorexia, bleeding, abdominal pain, convulsions, even depression, excessive nervousness or abnormal behaviour. In the cases of stronger intoxications, mycotoxins can attach to the DNA and cause mutations resulting in infertility and weakly developed or sick children. In the worst cases, they can cause liver and kidney damage, liver cancer, coma and even death. Nowadays there is still no treatment for mycotoxin intoxications, the only known solution being diet and intense hydration [6].

From all the toxins from mould, the most common are: aflatoxins, ochratoxins, citrinin, ergot alkaloids, patulin, zearalenone and trichothecene. These could be identified in food such as cereal, nuts, coffee, cocoa, spices, oleaginous seeds, dried and fresh fruit [7]. Most frequently in samples of grapes and wine, we come across aflatoxins and ochratoxins. It is known that these are actually the most toxic mycotoxins because the "International Agency for Research on Cancer" (IARC) assigned aflatoxins to group I (compounds that can cause cancer in the human body) and ochratoxin $\mathrm{A}$ to group 2B (compounds that can possibly cause cancer in the human body) [8]. As well as that, according to assessments performed by Codex Alimentarius, approximately $15 \%$ of the total quantity of OTA that is ingested by the human body comes from wine [9].

Aflatoxins were discovered in 1900, in England, when approximately 120,000 birds, fed with Aspergillus flavus infested Brazilian nuts, had died. The disease, unknown at the time, was called "Turkey X". They analysed the food sample with thin layer chromatography (TLC) and identified a series of fluorescent compounds that were found responsible for the outbreak [10]. In 1962 the chemical compound derived from Aspergillus flavus was called "aflatoxin" [11] - "a" (Aspergillus) - "fla" (flavus) - toxin. 
<smiles>COc1cc2c(c3oc(=O)c4c(c13)CCC4=O)[C@H]1C=CO[C@@H]1O2</smiles>

aflatoxin B1<smiles>COc1cc2c(c3oc(=O)c4c(c13)CCOC4=O)[C@H]1C=CO[C@@H]1O2</smiles>

aflatoxin $\mathrm{G} 1$<smiles>COc1cc2c(c3oc(=O)c4c(c13)CCC4=O)[C@H]1CCO[C@@H]1O2</smiles>

aflatoxin B2<smiles>COc1cc2c(c3oc(=O)c4c(c13)CCOC4=O)[C@H]1CCO[C@@H]1O2</smiles>

aflatoxin $\mathrm{G} 2$
Figure 1. Chemical structures of aflatoxins.

This was analysed with TLC and on a plaque exposed to ultraviolet light it was observed that there are two compounds of different colour: blue and green. They were called "aflatoxin B" (blue) and "aflatoxin G" (green) [11]. The B1, B2, G1 and G2 compounds are the main types of aflatoxins. Besides these, there were identified two metabolites, M1 and M2 (from B1 and B2) [12] and approximately 20 other aflatoxins (P1, Q1, B2a, G2a, etc.), most of which being produced through the biotransformation of the main metabolites [13]. From the total content of aflatoxins, $60 \%-80 \%$ are $\mathrm{B} 1$ and $\mathrm{B} 2, \mathrm{G} 1$, G2 do not usually appear without B1.

The main mouldy species that produce aflatoxins are Aspergillus (A. flavus, A. parasiticus and A. nomicus) but also Penicillium, Rhizopus, Mucor and Streptomyces. It was observer that the incidence of development of the Aspergillus flavus species is lower in areas with temperatures smaller than $20^{\circ} \mathrm{C}$, than in the areas with a warm climate with temperatures greater than $25^{\circ} \mathrm{C}$, and in some areas, the infection occurs only when the temperatures rise in association with drought [4]. The B1 aflatoxin generally needs higher temperatures $\left(24-28^{\circ} \mathrm{C}\right)$ than $\mathrm{G} 1\left(23^{\circ} \mathrm{C}\right)[10]$.

From a chemical point of view, aflatoxins (Fig. 1) are thermally stable compounds, but they act as an reactive in environments with $\mathrm{pH}$ less than 3 and are soluble in water and in organic solvents (methanol, acetonitrile, chloroform) [15].

Ochratoxins were first isolated in 1965 in South Africa. Initially, it was discovered that they come from Aspergillus species (A. melleus, A. muricatus, A. petrakii, A. sulphureus, A. albertensis şi A. alliaceus, A. Negri, but especially Aspergillus carbonarius), but they can also come from Penicillium verrucosum and Penicillium veridicatum $[3,9]$. Among the members of this mycotoxin class, ochratoxin A (OTA) is the most known and important due to its high toxicity [3].

Regarding the presence of ochratoxins in oenology, about $10 \%$ of the mould developed on grapes that are in the "veraison" stage can produce OTA, while when the grapes are fully maturated $47 \%$ of the mould can produce OTA. Regarding the type of wine, it was found that there is a<smiles>O=C(NC(Cc1ccccc1)C(=O)O)c1cc(Cl)c2c(c1O)C(=O)OCC2</smiles>

ochratoxin A

Figure 2. Chemical structure of ochratoxin A (OTA).

greater incidence of contamination for red wine than for white wine [3]. The wine making technique also has an influence on the OTA quantity, because in the maceration process the quantity of the toxin increases [9].

As a chemical compound, OTA (Fig. 2) is soluble in water and partially soluble in organic solvents (chloroform, ethanol or methanol) and in the presence of light and air, it is relatively unstable.

Taking into account the influence of these kinds of toxins on wine and human health, the current trends are to find a fast and efficient method of detecting these mycotoxins in order to protect the quality of wine. In Europe, the maximum quantity of aflatoxins and OTA allowed in wine and must, according to EU regulations is $2 \mathrm{ppb}$ [16]. Therefore, the method must be very sensitive as well as have thresholds of detection and quantification as low as possible.

\section{Experimental}

\subsection{Chemicals, materials and samples}

Standard of ochratoxin A, aflatoxins B1, B2, G1, G2, were acquired LGC standards and formic acid, organic solvents (HPLC grade) methanol and acetonitrile were obtained from Sigma-Aldrich. The ultra-pure water used for mobile phase preparation was purified through a Fistreem Cyclon coupled to a TermoScientific GenPure UV-TOC. Other chemicals and used materials were of analytical grade. Wine samples were from local didactical farm of the University of Agricultural Sciences and Veterinary Medicine "Ion Ionescu de la Brad" Iaşi NE region of Romania. Grapes samples of were proceed by the traditional fermentation method for wines technology. After gravity settler was done seeding with selected yeasts Saccharomyces cerevisiae. Alcoholic fermentation is finalized the wine was clarified with bentonite and then bottled. All samples were kept at $12{ }^{\circ} \mathrm{C}$ before opening the bottle and analysis.

\subsection{Chromatographic LC system}

The chromatography equipment was a Shimadzu Nexera system used for method development and validation. Tree pumps module Nexera X2 LC-30AD in binary configuration and one LC-20AT (Prominence series) for washing and maintenance of pressure in columns. All pumps have DGU-20A5R mobile phase degassers. Samples were introduced with Nexera X2 SIL-30 AC autosampler connected to a FCV-32AH valve for large volume injection and bypass for standard injection. Detection of analytes is done with fluorescence detector RF-20A XS (Prominence series) were also used and confirmation true a Nexera X2 SPD-M30A with HS cell 
of $85 \mathrm{~mm}$. The chromatography and extraction columns were thermostatted in a Nexera X2 CTO-30A column oven and mobile phase flow direction was changed by using an FCV-32AH high pressure column switching valve. The HPLC system was controlled by a CBM20A communication module. The data evaluation and acquisition of chromatography analyses were performed by Lab-Solution software v 5.75.

\subsection{Preparation of stock solution and wine samples}

Standard stock solutions of OTA $10 \mu \mathrm{g} / \mathrm{mL}$, aflatoxins $\mathrm{B} 1, \mathrm{G} 1,2 \mu \mathrm{g} / \mathrm{mL}$ and $0.5 \mu \mathrm{g} / \mathrm{mL} \mathrm{B} 2, \mathrm{G} 2)$ were utilised. Standard stock solution was stored at $-20^{\circ} \mathrm{C}$ in the dark. The calibration standard solutions were diluted in the concentration range from 1 to $200 \mathrm{ppb}$ (OTA), 1 to 100 (aflatoxins) using six calibration solutions.

Undiluted and diluted wine samples were filtered through a $0.22 \mu \mathrm{m}$ nylon filter into glass vials. A $50 \mu \mathrm{L}$ volume of filtered wine solutions was injected directly into the on-line SPE-LC system. All beer wine samples and working standard solutions were prepared fresh daily and injected in triplicate.

\subsection{LC column switching procedure}

The simultaneous wine sample extraction and mycotoxin determinations were performed using the on-line SPE- LC system. The SPE column used for mycotoxins extraction from wine samples was a Kinetex $5 \mu \mathrm{m}$ PFP $100 \AA$ column $(50 \times 4.6 \mathrm{~mm})$, particles core-shell silica (Phenomenex), with a washing mobile phase of $1 \%$ aqueous formic acid $\mathrm{pH} 2.15$ at a flow rate of $1 \mathrm{~mL} / \mathrm{min}$ and temperature of $40^{\circ} \mathrm{C}$. A $50 \mu \mathrm{L}$ volume of the filtered wine sample was directly injected in to the guard column where mycotoxins were retained while other polar interferences were washed out to the waste. The valve switch after 2.2 minute and extracted mycotoxins was transferred from the SPE column to the analytical column in the same elution direction.

The chromatographic separation of aflatoxins and OTA from interfering matrix components of the wine was achieved on the fused-core column Kinetex $1.7 \mu \mathrm{m}$ XBC18 $100 \AA$ column $(30 \times 3.0 \mathrm{~mm})$ (Phenomenex), using a mixture of $80 \%$ acetonitrile (solvent $\mathrm{A}$ ) and a solution of $1 \%$ formic acid in water, $\mathrm{pH} 2.15$ (solvent $\mathrm{B}$ ) at a flow rate of $2.5 \mathrm{~mL} / \mathrm{min}$ and a column temperature of $40^{\circ} \mathrm{C}$. A gradient elution was applied on the analytical column, with the initial conditions (solvent A/ B 0.1:99.9) held for $0.2 \mathrm{~min}$. Between 0.2 and $4.5 \mathrm{~min}$ of the chromatographic run the percentage of solvent A was linearly increased to $99.9 \%$. Thereafter, the percentage of solvent A was decreased to $0.1 \%$ in $90 \mathrm{~s}$, while the valve was switched back to the on-line SPE column position and the analytical column was equilibrated for $1 \mathrm{~min}$ to the initial conditions during the next sample pre-treatment step. Fluorescence excitation/emission detection wavelengths were set at $365 / 455 \mathrm{~nm}$ for aflatoxins and 333/460 $\mathrm{nm}$ for OTA.

\section{Results and discussion}

Wine is a complex matrix, which contains water, phenolic compounds, sugars, alcohols, acids, aldehydes, ketones, esters, nitrogen substances, sulphur compounds, mineral substances, stilbenes, bioactive substances and others. The ethanol as a fermentation product can partially affect the extraction of polar mycotoxins from the water because of early elution during the adsorption step. From these reasons, the procedure for extraction of mycotoxins from wine samples should be optimized and is due to cumbersome stapes that the human operator can influence. There is a good compatibility between online extraction (SPE) methodology and separation (LC or UHPLC) steps that must be taken into account. Therefore, the parameters of sample pre-treatment such as conditions of both mobile phases and stationary phases for extraction and separation were optimized before the method applied to wines sample analysis. The main aim of the work was to show a simple and modern analytical tool for the reliable determination of mycotoxins at trace levels in wine.

\subsection{Optimization of on-line SPE extraction step}

In this part we present the methodology which allowed fast extraction of mycotoxins from wine matrix with preconcentration on a first short column used to simulate SPE conditions. We utilised first a SPE $20 \times 2.0 \mathrm{~mm}$ $20 \mu \mathrm{m}$ particles commonly available reversed-phase C18, but the results are not satisfying.

By utilising Kinetex $5 \mu \mathrm{m}$ PFP $100 \AA$ column $(50 \times$ $4.6 \mathrm{~mm}$ ) we have improve the preconcentration of mycotoxins from wine samples with respect to the physicochemical properties (pKa) and lipophilicity (log P) of the analysed mycotoxins. OTA and aflatoxins are weak organic acids. The pKa values of OTA are in the ranges 4.2-4.4 and 7.0-7.3, respectively, for the carboxyl group of the phenylalanine moiety and the phenolic hydroxyl group of the isocoumarin part. The extracted mycotoxins should be in their neutral form to be well retained by hydrophobic interactions. Therefore, for sufficient retention of the mycotoxins of interest on the reversed-phase sorbent, the washing mobile phases were acidified with $1 \%$ formic acid to $\mathrm{pH}$ 2.15. A compromise between interference removal and the sufficient recovery of mycotoxins must be found during washing solution optimization. Therefore, the influence of the washing mobile phase on the SPE procedure was studied to ensure the fast and quantitative transfer of interfering matrix for disposal and simultaneous retention of the target analytes. The key parameters of extraction are the lipophilicity of the analytes, and the chemistry and extraction capacity of the SPE precolumn. The range of washing mobile phase ratios tested was from 0.1 to $40 \%$ of acetonitrile ( $80 \%$ ) with $1 \%$ aqueous formic acid $\mathrm{pH} 2.15$. When the percentage of pure acetonitrile exceeded $20 \%$ the more polar aflatoxins (G2, B2, G1 and B1) can be lost during elution of wine matrix. Therefore, the mobile phase with acetonitrile range from 0.1 to $35 \%$ was utilise. After $2.2 \mathrm{~min}$ of gradient washing at a flow rate of $2.5 \mathrm{~mL} / \mathrm{min}$ we switch to first column and connected with the second column that is kept with $1 \%$ aqueous formic acid $\mathrm{pH} 2.15$ at $1 \mathrm{~mL} / \mathrm{min}$ by the third pump (20AT). Since the aim of the experiment is to have a short analyse time, long washing periods were not implied so to improve clean-up efficiency. A second valve switch was performed a soon as the OTA is eluted from second column and is dove in order to minimise the pulse of rotation valves. These on-line SPE conditions produced a 


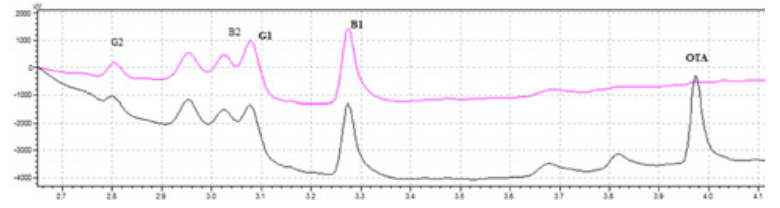

Figure 3. Spectral separation on DAD at $370 \mathrm{~nm}$ for aflatoxins and $345 \mathrm{~nm}$ for OTA.

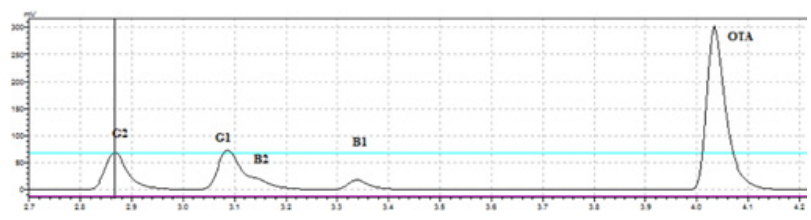

Figure 4. Detection of florescence for all toxins.

satisfactory elution of interfering compounds to the waste and sufficient resolution between peaks. The efficiency of the on-line sample pre-treatment procedure is depicted on the chromatogram in Figs. 3 and 4.

Increasing the volume of the injected sample is an effective way to improve the sensitivity of the on-line SPE-LC method. Nevertheless, the breakthrough volume of the SPE column resulting in analytes elution should not be exceeded. Therefore, compromise must be found between the washing of the residual peaks of the matrix using a larger injected sample volume and efficiency of the clean-up step. Injection volumes larger than $500 \mu \mathrm{L}$ were not tested in our study because the residual peak of matrix interferences complicated the determination of the aflatoxins peaks (especially B2 and G1). Long isocratic washing step time did not lead to a significant elimination of the ballast matrix peak. Therefore, a compromise between the sensitivity and selectivity of the extraction step was found at $50 \mu \mathrm{L}$ of undiluted wine which was injected directly into the extraction column.

\subsection{Chromatographic separation}

Fused-core technology is based on semi-porous particles instead of the traditional totally porous ones. In our study, fused-core particle columns with dimensions of $30 \times 3.0 \mathrm{~mm}$ and particle size of $1.7 \mu \mathrm{m}$ with stationary phases Pentafluorophenyl PFP (Phenomenex) is utilise for optimal separation time after on-line SPE extraction. Mobile phases containing mixtures of aqueous acetonitrile $(80 \%)$ with a $1 \%$ formic acid solution in water, $\mathrm{pH} 2.15$ in the range from 0.1 to $99.9 \%$ for optimal separation. The column oven temperature was set at $40^{\circ} \mathrm{C}$ as a compromise between lowering the back-pressure in the system and stationary phase temperature stability. Other consideration is regarding the spikes that can appear randomly in to the control temperature detection system. Both detectors operate at $30^{\circ} \mathrm{C}$.

In order to optimize the separation conditions in the on-line SPE system, the gradient elution was tuned for better resolution of the aflatoxins peak from the residual peak of interferences or the valve switch. The time of the valve switch was set to 2.2 minute. The gradient elution program started at this moment from $0 \%$ of acetonitrile, so the lower content of acetonitrile at the beginning of the separation after the flow switch from the extraction column to the analytical column enabled gradual reabsorption of analytes at the beginning of the chromatogram. Thus, the peak of OTA was eluted over a longer time and well separated from the aflatoxins and wine matrix. A gradient elution was applied on the both analytical columns, with the initial conditions at switch over and its continuation from $35 \%$ eluent A (80\%). Between 2.2 and 4 min of the chromatographic run, the percentage of solvent A was linearly increased to $99.9 \%$. Thereafter, the percentage of solvent A kept for 90 seconds and was decreased to $0.1 \%$ over $60 \mathrm{~s}$, while the valve was switched back to the loading SPE position and the analytical column was equilibrated for $1 \mathrm{~min}$ to the initial conditions during for the next sample SPE injection. Under the optimal conditions all five toxins were eluted selectively with some matrix interferences in less than 7 min including the sample pre-treatment step.

\subsection{Validation of on-line SPE-HPLC method}

The validation and analytical performance of the proposed method was tested in terms of linearity, limits of detection (LOD), limits of quantification (LOQ) and accuracy (recovery). Standard solutions were injected five times into the chromatographic system. Results are summarized in Table 1. Calibration curves were measured for standard solutions at six concentration levels, depending on the fluorescence intensity of each mycotoxin. The linearity for standard were measured in the range of 1 to $200 \mathrm{ppb}$ for OTA also 1 to 100 for aflatoxins G2, B2, G1 and $\mathrm{B} 1$. Under the validated conditions, linear relationships with perfect regression coefficients both in standard and matrix calibration curve were found in the range 0.985-0.998 for all toxins. Linear regression parameters determination for standard calibrations are described by the regression equations (slope and intercept). Limits of detection and quantification, calculated as the minimum toxin concentration yielding a signal to noise ratio equal to three and ten times the standard deviation $(\sigma)$.

LOQs were established as low as $0.15 \mathrm{ppb}$ for OTA and the highest is $5.32 \mathrm{ppb}$ for G2, one cause is the signal registered in from of B2. The obtained LOQ values are low enough to evaluate mycotoxins content. The percentage recovery values were calculated as (measured area of spiked placebo beer sample with standard solution/peak area of standard solution at the same concentration) $\times 100 \%$. The recovery values were found to be in the range $83.18-109.42 \%$ for toxins. Values are relatively close to $100 \%$ indicate that there are no interferences or other matrix suppression effects. The on-line SPE-LC system was found to be very sensitive, selective and rugged for the analysis of filtered wine samples without additional sample pretreatment. The values of RSD for retention times, peak symmetry and chromatography resolution were not higher than $5 \%$ at the beginning and the end of the study.

\subsection{Analysis of wine}

In this work, the analysed samples were divided into four categories: dry white wine, wine demi sweet, wine red dry and red wine maturated in barriques. Twelve wine samples are analysed, ten from 2016 year of harvest and tow are from 2015. All samples have OTA levels below the maximum permitted limits of $2 \mathrm{ppb}$ (summarized in Table 2). The results confirmed the presumption that the 
Table 1. Analytical characteristics of the validated online SPE-LC method.

\begin{tabular}{|l|c|c|c|c|c|}
\hline & G2 & B2 & G1 & B1 & OTA \\
\hline Standard linear calibration range (ppb) & $5-100 \mathrm{ppb}$ & $5-100 \mathrm{ppb}$ & $1-100 \mathrm{ppb}$ & $1-100 \mathrm{ppb}$ & $1-200 \mathrm{ppb}$ \\
\hline Slope & 16844.6 & 273.72 & 74.096 & 1028.79 & 3192.83 \\
\hline Intercept & 35153.2 & 932.83 & 1610.85 & 7456.47 & 154688 \\
\hline Regression coefficient $\left(\mathrm{r}^{2}\right)$ & 0.98553 & 0.90239 & 0.98545 & 0.98554 & 0.9982 \\
\hline LOD (ppb) & 0.59 & 0.93 & 1.61 & 1.14 & 0.15 \\
\hline LOQ (ppb) & 1.95 & 3.08 & 5.32 & 3.76 & 0.51 \\
\hline Precision (RSD, \%) & 2.01 & 1.17 & 3.9 & 2.17 & 4.07 \\
\hline Accuracy for spike recovery (\%) & 95.71 & 94.58 & 85.18 & 113.24 & 109.42 \\
\hline
\end{tabular}

Table 2. Occurrence of ochratoxin A and aflatoxins in the some Romanian wine samples.

\begin{tabular}{|c|c|c|c|c|c|c|c|}
\hline Grape sort & year of harvest & wine type & G2 (ppb) & B2 (ppb) & G1 (ppb) & B1 (ppb) & OTA (ppb) \\
\hline Zghihara & 2016 & dry white & $\mathrm{D}$ & ND & ND & $3.33 \pm 0.01$ & $\mathrm{D}$ \\
\hline Agiloté & 2016 & dry white & $\mathrm{D}$ & ND & ND & $2.77 \pm 0.01$ & $\mathrm{D}$ \\
\hline Fetească albă & 2016 & dry white & $\mathrm{D}$ & ND & ND & $11.09 \pm 0.01$ & $\mathrm{D}$ \\
\hline Fetească regală & 2016 & dry white & $\mathrm{D}$ & ND & ND & $\mathrm{D}$ & ND \\
\hline Sauvignon blanc & 2016 & dry white & $\mathrm{D}$ & ND & ND & $\mathrm{D}$ & ND \\
\hline Traminer & 2016 & demi sweet & $\mathrm{D}$ & ND & ND & $18.15 \pm 0.02$ & $\mathrm{D}$ \\
\hline Chadornnay & 2016 & demi sweet & $\mathrm{D}$ & ND & ND & $14.27 \pm 0.02$ & $\mathrm{D}$ \\
\hline Fetească neagră & 2016 & red dry & $1.72 \pm 0.01$ & ND & ND & $11.71 \pm 0.02$ & $\mathrm{D}$ \\
\hline Merlot & 2016 & red dry & $4.67 \pm 0.01$ & ND & ND & $22.95 \pm 0.03$ & $\mathrm{D}$ \\
\hline Cabernet Sauvignon & 2016 & red dry & $2.46 \pm 0.01$ & ND & ND & $22.19 \pm 0.03$ & $\mathrm{D}$ \\
\hline Merlot & 2015 & red barrique & $1.88 \pm 0.01$ & ND & ND & $28.12 \pm 0.03$ & $\mathrm{D}$ \\
\hline Cabernet Sauvignon & 2015 & red barrique & $\mathrm{D}$ & ND & ND & $\mathrm{D}$ & ND \\
\hline
\end{tabular}

risk of OTA occurrence in wines is very low. Among the samples at 4 wines we find contamination by aflatoxin G2 with concentrations lower than $5 \mathrm{ppb}$, which is a low level. Other samples have present this aflatoxin but under the detection limit and it was evidence only by DAD detector. For the aflatoxin B2 and G1 we didn't found any amount.

In the case of B1 at a number of samples we found a fairly concentration and in the rest of the samples some quantity is found but cannot be confirmed by spiking or complementary detection technique. All samples analysed are not commercial wines and aim of the study is to evaluate the method and state health of the wines.

\section{Conclusion}

The method developed is an alternative approach for sample preparation and separation of five mycotoxins in wine. This new method for on-line solid-phase extraction of ochratoxin A and aflatoxins from wine samples is optimized fast and reproducible. Utilization of on-line SPE sample pretreatment with a relatively large volume sample injection was shown to be the method of choice for fast determination of very low concentrations of mycotoxins in wines. The online SPE-UHPLC with dual detection (DAD-FLD) for confirmation showed high sample throughput, low solvent consumption and high sample preconcentration of mycotoxins depending on the injected volume. The combination of sensitive fluorescence detection and a highly efficient chromatography process on both fused-core columns together with sample preconcentration resulted in perfect sensitivity of the developed method. The limit of quantification for OTA at least able to determine maximum permitted limits for
OTA occurrence. Aflatoxin B1 is present in all samples in varying amounts.

This work was carried out by the Partnership in priority areas PN II, developed with the support of MEN - UEFISCDI, project no. 183/2014 (PN-II-PT-PCCA-2013-4-0333) “Technology of capitalization of the bioactive elements from the grape seed waste with usefulness in the food and pharmaceutical industry, plant and environmental protection (Acronym: PROVITIS)".

\section{References}

[1] FAO-OIV 2016 http://www.oiv.int/public/ medias/ 4911/fao-oiv-grapes-report-flyer. pdf

[2] A. Rahmani, S. Jinap, F. Soleimany, Comprehensive reviews in Food Science and Food Safety 8, 202-251 (2008)

[3] S.M. Jović, A.V. Petrović, N.R. Marković, Proc. Nat. Sci, Matica Srpska Novi Sad 116, 91-100 (2009)

[4] P.J. Cotty, L.S. Lee, National Cotton Council of America, Las Vegas, NV, 34-36 (1990)

[5] R. Jaime-Garcia, P.J. Cotty, Phytopathology 93, 1190-1200 (2003)

[6] J.W. Bennett, Klich M., Clin Microbiol Rev. 16(3) 497-516 (2003)

[7] I. Lhotská1, D. Šatínský1, L. Havlíková1, Petr Solich, Anal Bioanal Chem. 408, 3319-3329, (2016)

[8] IARC, 2016: http://monographs . iarc.fr/ENG/ Classification

[9] S. Somma, G. Perrone, A.F. Logrieco, Phytopathologia Mediterranea 51(1), 131-147 (2012)

[10] B.I. Agag, Ass. Univ. Bull. Environ. Res. 7, 1, March (2004) 
[11] K. Sargeant, R.B. Carraghan, R. Allcroft, Chem. and Ind., 53-55 (1963)

[12] D.S. Patterson, E.M. Galaney, B.A. Roberts, Food and cosmetic toxicology 16, 49-50 (1978)

[13] J.G Heathcote., J.R. Hibbert, Elsevier Scientific Publishing Company, Amsterdam, The Netherlands (1978)
[14] M. Weidenborner, Encyclopedia of food mycotoxins, Spronger Publisher Berlin, New York, London (2001)

[15] V.D. Cotea, C. Zănoagă, V.V. Cotea, Tratat de Oenochimie, Editura Academiei Române, II, 103 (2009)

[16] OIV Resolution CST 1/2002 